\title{
Spectral biometrical recognition of fingerprints
}

\author{
Tibor Csongrády*, Elena Pivarčiová ${ }^{\dagger}$ \\ Department of Informatics and Automation Technology, Technical University in Zvolen, \\ Masarykova 24, Zvolen, 960 53, Slovakia
}

Received 29 Mar 2011 ; accepted 01 Jun 2011

\begin{abstract}
The article is aimed at fingerprint recognition or verification, which is used mainly in access control systems. The proposed method of fingerprint verification uses image registration (reconciliation) by applying the Fourier-Mellin transform and consequently compares images by the Fourier transform using modified phase correlation.

Keywords: biometry • biometric systems • Fourier-Mellin transform

(c) Versita Sp. z o.o.
\end{abstract}

\section{Introduction}

Biometrics is the science dealing with unique and measurable characteristics and properties to recognize and verify a person's identity based on physiological and behavioral characteristics. A special sub-part of biometrics is behaviometrics which observes properties (not physical parameters) of a human, learnt or obtained during the life of a person.

Biometrics is largely used for automatic prevention of unauthorized accesses or unwanted usage of bank cards, cell phones, desktops, workstations or computer networks. Access to domains, log-in to computer networks, log-in to applications, protection of data, remote access to sources and electronic transactions are some of the other areas of the technologies' utilization. Other significant applications are access management to structures, attendance systems and identity control at the borders or identification of a bank card owner paying or withdrawing cash.

The article deals with one of the biometric characteristics: fingerprints. A person's identification by fingerprints is the best known and one of the most applied biometric methods to identify or verify personal identity. The article focuses on fingerprint recognition or verification which is used mainly in access control systems.

Some of the classical methods of fingerprint recognition are those where a grey-scale image of fingerprint is firstly preprocessed (filtration, segmenting, thinning) and then some of the characteristic features (minutiae) are extracted for the recognition, for example (see Ref. [6]). Recognition is made on the basis of information about the features position and further information is obtained from the modified image. These methods are sensitive to noise and deformations. In the preprocessing, errors can arise, making the recognition more difficult. An alternative approach can be represented by methods which do not require preprocessing, e.g. recognition according to fractals, according to the Fourier spectrum (see Ref. [8]), or according to mutual information (see Ref. [3]), etc.

*E-mail: cso@vsld.tuzvo.sk

+ E-mail: epivarci@vsld.tuzvo.sk (Corresponding author) 


\section{Two-dimensional discrete Fourier transform}

The discrete Fourier transform (DFT) is defined as (see Ref. [1]):

$$
F(u, v)=\sum_{x=0}^{M-1} \sum_{y=0}^{N-1} f(x, y) \mathrm{e}^{-j 2 \pi\left(\frac{u x}{M}+\frac{v y}{N}\right)}=F\{(x, y)\}
$$

where $x, y$-pixel position, $f(x, y)$ - pixel value, $M, N$ - image dimensions, $u, v$ - spatial frequencies $(u=0,1, \ldots, M-1$ and $v=0,1, \ldots, N-1), j-$ imaginary unit.

Inverse Discrete Fourier transform (IDFT):

$$
f(x, y)=\frac{1}{M N} \sum_{u=0}^{M-1} \sum_{v=0}^{N-1} F(u, v) \mathrm{e}^{j 2 \pi\left(\frac{u x}{M}+\frac{v y}{N}\right)}
$$

for $x=0,1, \ldots, M-1$ and $y=0,1, \ldots, N-1$.

Function $F(u, v)$, obtained by the Fourier transform, is a complex function and can be expressed as (see Ref. [1]):

$$
F(u, v)=R(u, v)+j l(u, v)=|F(u, v)| \mathrm{e}^{-j \phi(u, v)},
$$

where $R(u, v)$ is real and $I(u, v)$ is an imaginary component of the function $F(u, v),|F(u, v)|=\sqrt{R^{2}(u, v)+I^{2}(u, v)}$ is an amplitude frequency spectrum of the function $F(u, v)$ and $\phi(u, v)=\tan ^{-1} \frac{(u, v)}{R(u, v)}$ is a phase spectrum. In Fig. 1 amplitude and phase spectrum of the Fourier transform of a fingerprint image is shown.
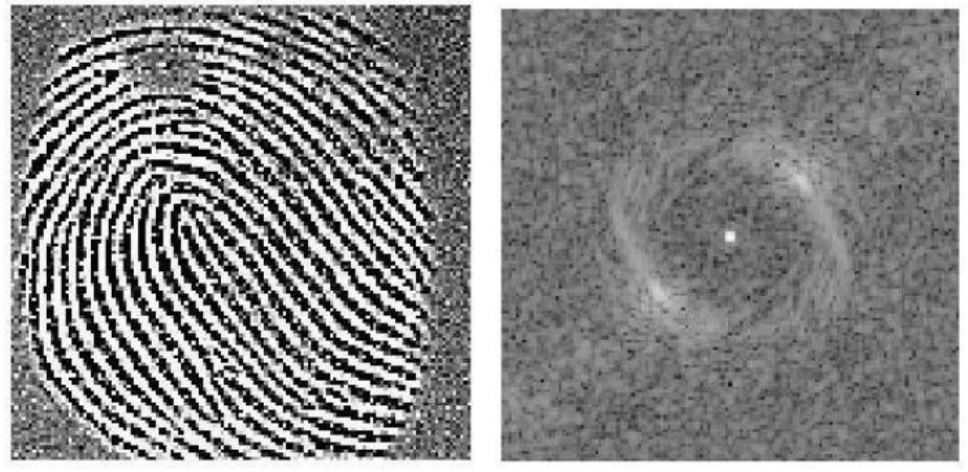

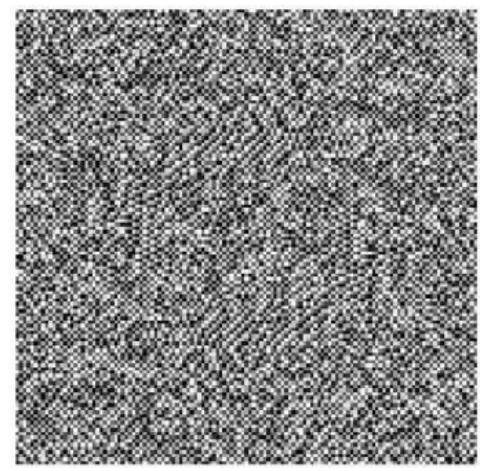

b

Figure 1. Fingerprint image and its Fourier transform. a) amplitude spectrum b) phase spectrum.

\subsection{Some properties of the discrete Fourier transform}

\subsubsection{Invariance of the Fourier transform towards a shift}

If the spatial function is shifted by a constant, then this shift is expressed as a constant shift in phase DFT only (see Ref. [1]):

$$
\begin{aligned}
& f(x, y) e^{j 2 \pi\left(\frac{u_{0} x+v_{0} y}{N}\right)} \leftrightarrow F\left(u-u_{0}, v-v_{0}\right), \\
& f\left(x-x_{0}, y-y_{0}\right) \leftrightarrow F(u, v) e^{-j 2 \pi\left(\frac{u x_{0}+v y_{0}}{N}\right)}
\end{aligned}
$$


The shift does not influence the amplitude:

$$
\left|F(u, v) e^{-j 2 \pi\left(\frac{u x_{0}+v y_{0}}{N}\right)}\right|=|F(u, v)| .
$$

Fourier-Mellin transform uses invariance of DFT amplitude towards the shift. In Fourier-Mellin transform scale factor and rotation are shown as shifts, which are then removed by the second DFT for amplitudes which are invariant towards the shift (see Ref. [10]).

\subsubsection{Achieving invariance towards rotation}

The Fourier transform of a two-dimensional function is not invariant towards a rotation. Fourier image of a rotated fingerprint by $\theta$ function will also be rotated by the same angle.

Invariance of the Fourier transform towards rotation can be achieved by conversion of the shift in the function of one coordinate.

For such a conversion it is possible to use the fact that to express the function in polar coordinates rotation of an image $\theta$ is then manifested as a change of this particular coordinate. The function $f(x, y)$, defined in Cartesian coordinates $(x, y)$, can be defined in polar coordinates $(\theta, r)$ as function $f(\theta, r)$, where $r$ represents a radial component and $\theta$ an angle component (see Ref. [10]).

Fig. 2 shows that for projecting in polar coordinates the rotation is shown as the sum of two angle components of the original shift $\theta 0$ and a rotation angle $\Delta \theta$.

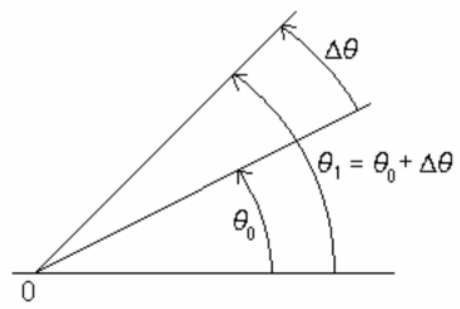

Figure 2. Rotation in polar coordinates.

The Fourier transform of polar function can eliminate rotation change in $\theta$ concerning amplitude, which is invariant towards a shift. Amplitudes of the Fourier transform of reversed and non-reversed image will be identical following this fact.

\subsubsection{Achieving invariance towards scale}

If the scale of the spatial function is adjusted by a constant, the Fourier transform shall have an inverse adjusted scale by the same constant.

The change of the scale in polar coordinates represents multiplication of radial component $r$ by the constant $k$, which characterises the scale change, as Fig. 3 shows.

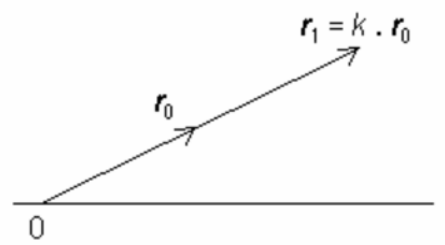

Figure 3. The scale in polar coordinates. 
If the images are shifted in Cartesian coordinate system, in which the result of DFT is also presented, mathematically it means adding the constant, characterizing this shift, to all original coordinates. When the scale is changed in polar coordinates, then the scale change is characterized by constant of multiplication $k$. If a logarithm for this multiplication is used, the operation of multiplying is transformed into the sum of $\log$ arithms: $\log k \cdot r_{0}=\log k+\log r_{0}$. Therefore application of the logarithm to radial component $k . r$ will transfer scale change into shift in logarithmic coordinates.

\section{Fourier-Mellin transform}

\subsection{Logarithmic-polar presentation}

As shown before, using the logarithmic-polar transformation it is possible to achieve both invariance towards a scale change and invariance towards a rotation.

Image function $f(x, y)$ can be sampled as function $f\left(\theta, \mathrm{e}^{r}\right)=f(\theta, \rho)$, where $r$ is distance from the centre of an image (see Fig. 4).

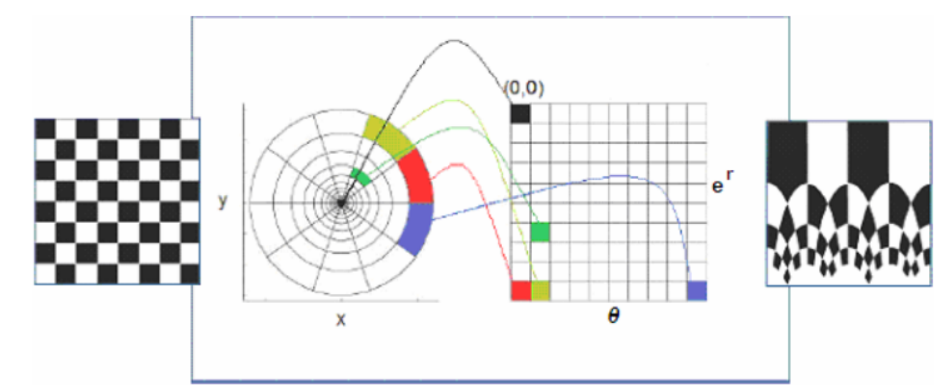

Figure 4. Logarithmic-polar transformation (see Ref. [4]).

Let us assume that the centre of the image represents starting point of transformation. Every pixel in the image can be represented as distance $r$ from the centre of the image and angle $\theta$. When the image is turned, only $\theta$ changes, $r$ remains the same.

If exponential scale $\log r$ is used instead of the representation of the second pixel coordinate as measure $r$, it is possible to convert the change of the scale into shift (see Ref. [10]).

If the image has the scale changed by $k$, Cartesian point $P(x, y)$ in the image will be represented in logarithmic-polar coordinates as $P(\theta, \log (k . r))$. Then point $P$ with a changed scale will be expressed as shift: $P(\theta, \log k+\log r)(\operatorname{see}$ Ref. [10]).

The change of rotation and scale is converted into a shift in the logarithmic-polar transformation.

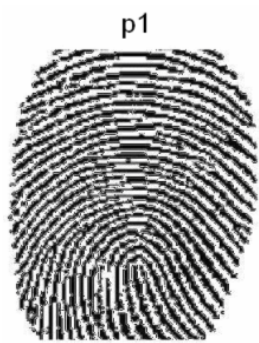

a

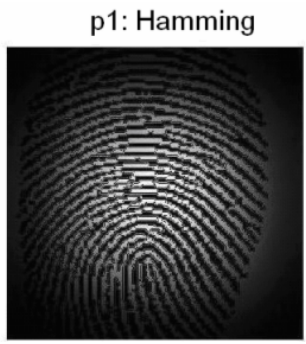

b

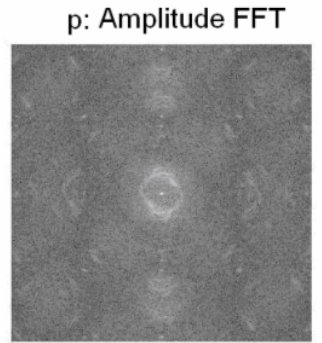

c

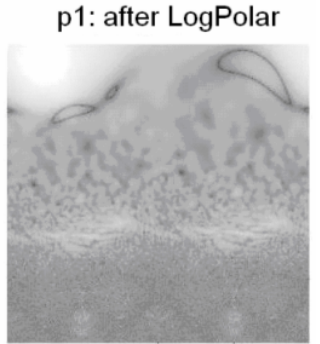

d

Figure 5. Fingerprint image: a) Original image, b) Hamming window, c) Fourier spectrum in Cartesian coordinates, d) Fourier spectrum in logarithmic-polar coordinates. 
Logarithmic-polar transformation allows to express the scale and rotation changes as shifts. These can be removed concerning the amplitude of the Fourier transform converted into logarithmic-polar coordinate system. This is the basis for the Fourier-Mellin transform (see Ref. [10]).

The Fourier transform from logarithmic-polar coordinates is equivalent to the Fourier-Mellin transform.

In Fig. 5 there is shown a fingerprint image and its Fourier spectrum in Cartesian and logarithmic-polar coordinates.

\subsection{Image registration using the Fourier-Mellin transform}

Registration (reconciliation, matching, alignment) is defined as finding the most suitable geometric transformation which describes mutual relations between reference and input data file.

Image registration allows maximal possible geometric alignment of corresponding points and objects in images (see Ref. [7]), i.e. identifies position and spatial orientation of these objects.

Image registration using the Fourier-Mellin transform allows reconciliation of images which are shifted, rotated and scaled (see Ref. [2, 9] (see the algorithm below)).

\section{Registered images comparison}

In next stage it was necessary to compare two fingerprint images: the reference one and the tested one, using suitable measures and comparing score to find out if they come from the same finger or not.

We used two different approaches:

a) comparison of images after registration (turned backwards, shifted, and with adjusted scale)

b) comparison of an amplitude and phase spectrum of the Fourier-Mellin transform.

The second approach of the solution of the fingerprint verification is comparison of fingerprint images by applying amplitude and phase spectrum of the Fourier-Mellin transform (without the necessity of registration) using their invariance towards a shift, rotation, and scale. Registration of images is used just for visual control of the comparison. As the Fourier-Mellin transform reduces mutual image rotation to the shift along the horizontal coordinate and the change of the scale to shift along vertical coordinate, we tried to compare directly these spectrums without the intermediate stage of registration. Two images, one of which is shifted and/or scaled, have identical amplitudes of the Fourier-Mellin transform.

The application of the "window" function to images which have considerably changed scale, rotation and/or shift (as shown in Fig. 6) does not have to be satisfactory to compare images therefore from every image there was cut an effective area from the middle of aligned fingerprints of half dimensions. This process can improve the precision of fingerprint comparison because not overlaying areas of two images usually contain noise components in POC function. In Fig. 6 there is a view of fingerprints after cutting the effective area from the registered fingerprints.

To evaluate the results of image comparison we used several traditional as well as own measures to calculate a comparing score which quantifies similarity between the tested and reference images. The calculation of measures was verified in various combinations: with/without the application of the Hamming window, with/without the application of the low-pass filter, with/without the application of pre-processing. From the comparison techniques being tested the best results were produced by modified phase correlation (MPOC, Modified Phase Only Correlation). The suggested weight function $W(u, v)$ has the shape of Laplace spectrum of Gaussian function (see Ref. [5]):

$$
W(u, v)=\left(\frac{u^{2}+v^{2}}{\alpha}\right) \mathrm{e}^{-\frac{u^{2}+v^{2}}{2 \beta^{2}}},
$$

where $u, v$ are 2D coordinates, $\beta$ is parameter which controls the function width and $\alpha$ is used for normalization. Parameters were applied for fingerprints testing $\beta=0.1$. $N$ and $\alpha=3.14$. $\beta$.

Thus modified phase-only function (MPOC) of images $g 1$ and $g 2$ is given:

$$
\tilde{q}_{g_{1} g_{2}}(x, y)=F^{-1}\left\{W(u, v) \frac{G_{1}(u, v) \overline{G_{2}(u, v)}}{\left|G_{1}(u, v) \overline{G_{2}(u, v)}\right|}\right\} .
$$




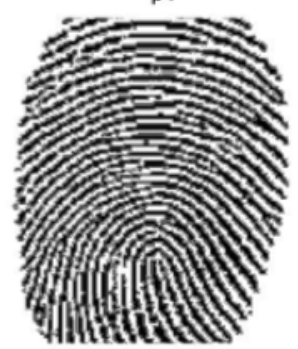

p1: Hamming

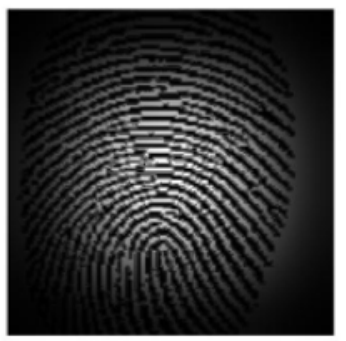

Pôvodné obrázky

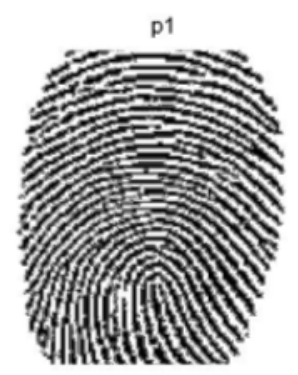

Vyrezané: p1

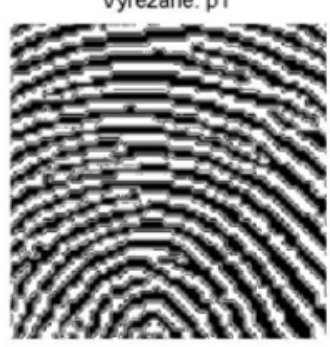

Obrázky po registrácii
p1-X-15Y-14M0.8R-5

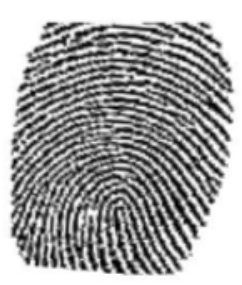

p1-X-15Y-14M0.8R-5: Hamming

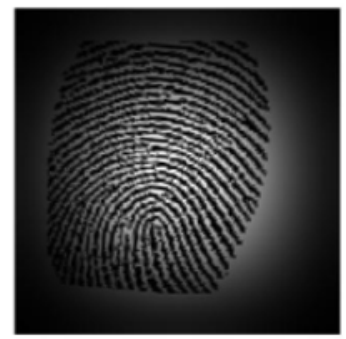

p1-X-15Y-14M0.8R-5 po registr.

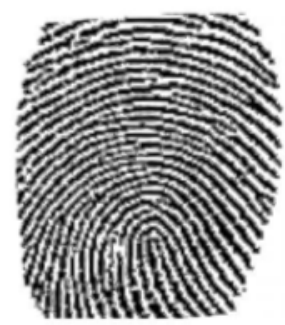

Vyrezané: p1-X-15Y-14M0.8R-5

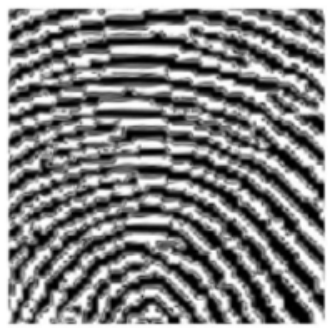

Figure 6. Selection of the effective area of images.

The peak value of the MPOC function $\tilde{q}_{g_{1} g_{2}}(x, y)$ is also invariant to the change of shift and brightness.

The peak value of the MPOC function according to (8) was used as a similarity measure for image matching: if two images are similar, their MPOC function will give a distinct sharp peak, if they are dissimilar, then the peak drops significantly.

POC and MPOC values comparison for similar images is in Fig. 7c. 

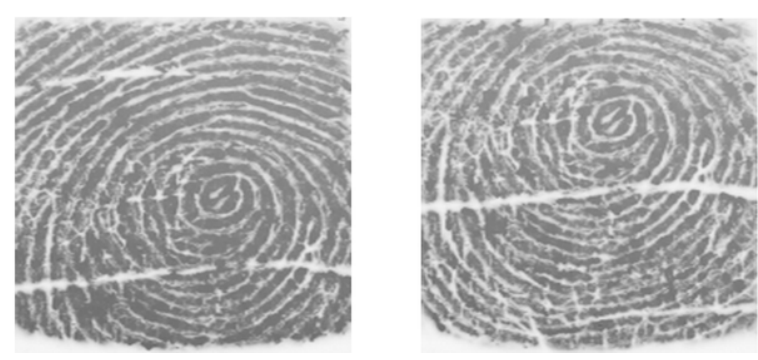

a

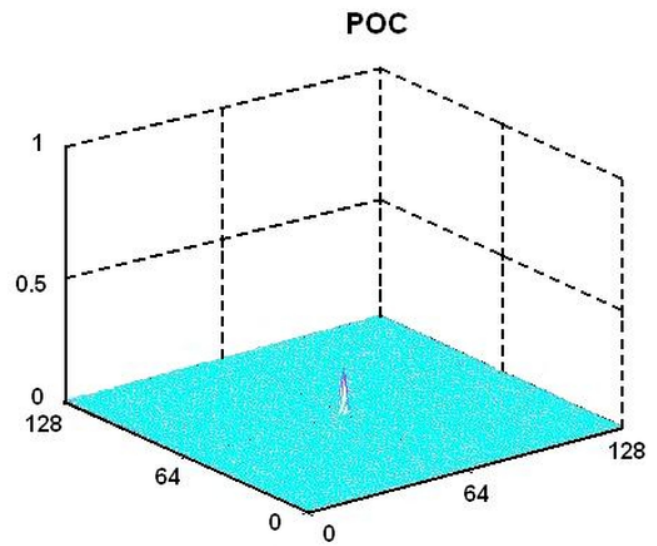

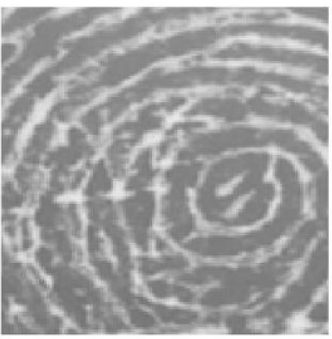

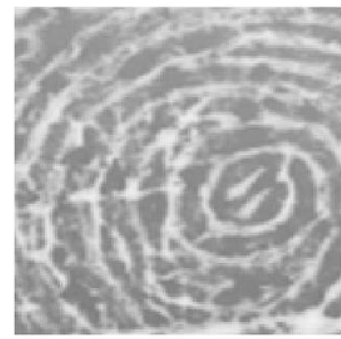

b

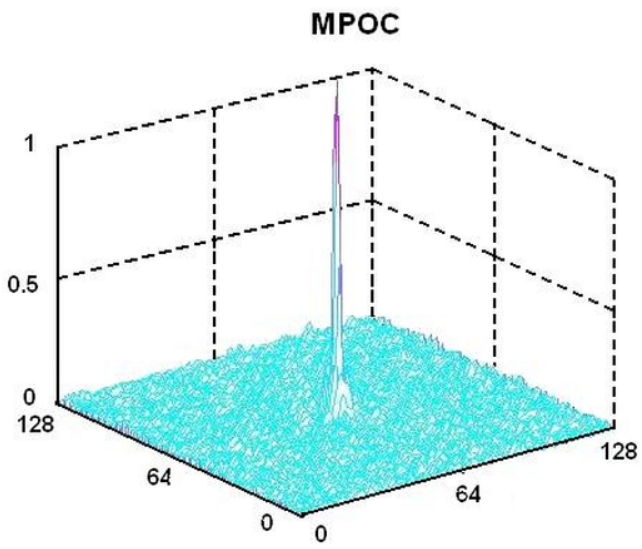

Figure 7. a) Original fingerprints $(256 \times 256$ pixels). Fingerprints after registration (cut-off centre of $128 \times 128$ pixels). Phase only correlation (POC) and modified phase only correlation (MPOC) between similar images.

\section{Proposed algorithm of fingerprint verification}

The proposed method of fingerprint verification uses image registration (reconciliation) using the Fourier-Mellin transform and consequently compares the images by the Fourier transform using the modified phase correlation (see Fig. 8).

\section{Achieved results}

In this experiment we scanned fingerprint of ten persons in the age range from 25 to 63 years: 7 males (including one twin pair) and 3 females. From every person there were scanned ten fingerprint images of the right thumb and ten fingerprint images of the left thumb. Fingerprints were taken at different times with at least one day time break. The images were in the resolution of $256 \times 256 \times 8$ pixels.

To scan fingerprints we used FPC-AM module of Fingerprint Cards AB company, which contains plane contact sensor FPC 1011C to scan fingerprints using reflection capacity technology. Active area of the sensor has the dimensions of $10.64 \mathrm{~mm}$ by $14 \mathrm{~mm}$, resolution 8 bits (256 on the gray level).

The false rejection test is to evaluate the False Rejection Rate (FRR), where FRR is the probability that an authorized person is falsely rejected. FRR represents number of fingerprints that were proclaimed as discordant ones although they belonged to the same persons considering total number of tested fingerprints that were supposed to be accepted (belonging to the same persons).

The total number of accepted fingerprint images used for the test of false rejection is 180 (see Tab. 1).

The false acceptance test is to evaluate the False Acceptance Rate (FAR), where FAR is the probability that a nonauthorized person is accepted as an authorized person. FAR represents number of fingerprints that were proclaimed as 


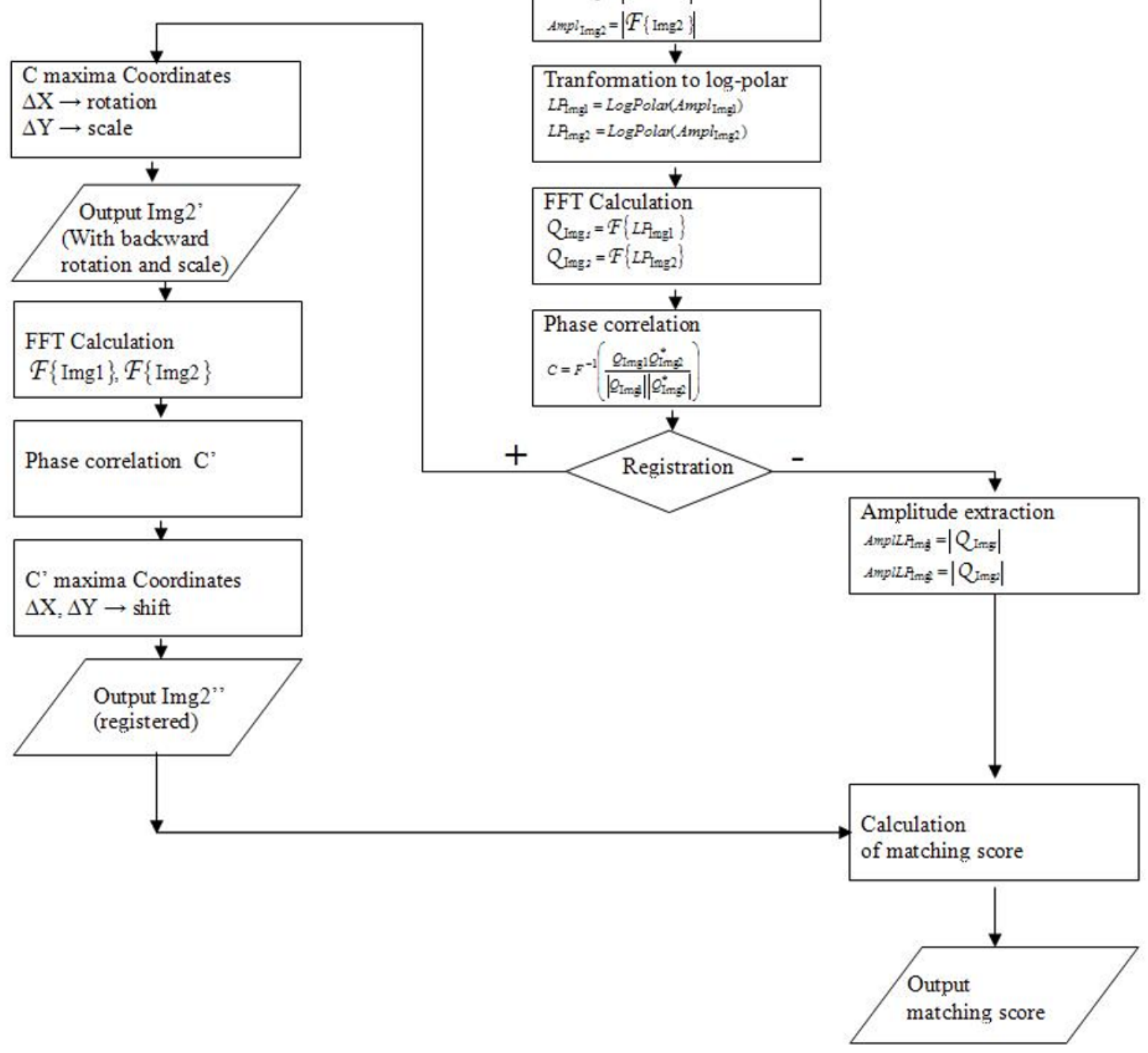

Figure 8. The diagram of the proposed algorithm. 
Table 1. Input values for FAR and FRR tests.

\begin{tabular}{|c|c|c|}
\hline Parameter & Value & Description \\
\hline Number of persons & $x=20$ & $\begin{array}{l}10 \text { persons by } 10 \text { fingerprints of the left thumb and } \\
10 \text { fingerprints of the right thumb }\end{array}$ \\
\hline Number of fingerprints from a person & $n=10$ & 1 specimen +9 prints \\
\hline Number of all prints & $y=x \cdot n=200$ & \\
\hline Number of all specimens & $v=x=20$ & \\
\hline Number of specimens from a person & $v_{1}=1$ & \\
\hline Number of prints to compare from a person & $z=n-v_{1}=9$ & \\
\hline Number of all tests & $S=v \cdot x \cdot z=3600$ & Comparison of all prints with the specimens \\
\hline Number of accepted tests from a person & $A_{1}=z \cdot v_{1}=9$ & \\
\hline Number of non-accepted tests from a person & $R_{1}=\left(v-v_{1}\right) \cdot z=171$ & \\
\hline Number of all accepted tests & $A=x \cdot A_{1}=180$ & \\
\hline Number of all non-accepted tests & $R=x \cdot R_{1}=3420$ & \\
\hline
\end{tabular}

identical ones although they belonged to different persons considering total number of tested fingerprints, that were not supposed to be accepted (belonging to different persons).

The total number of fingerprint images for False Acceptance Rate is 3420 (see Tab. 1).

To calculate biometric system errors of FAR and FRR there were used input values provided in the Tab. 1.

Equal Error Rate (EER), which is defined as the error rate where the FAR and the FRR are equal, was applied to numeric comparison of individual algorithms. The lower value of EER, the more accurate the system is.

The performance of the biometrics system was evaluated by Receiver Operating Characteristic (ROC) curve, which illustrates the FAR in dependence on the FRR for different threshold values.

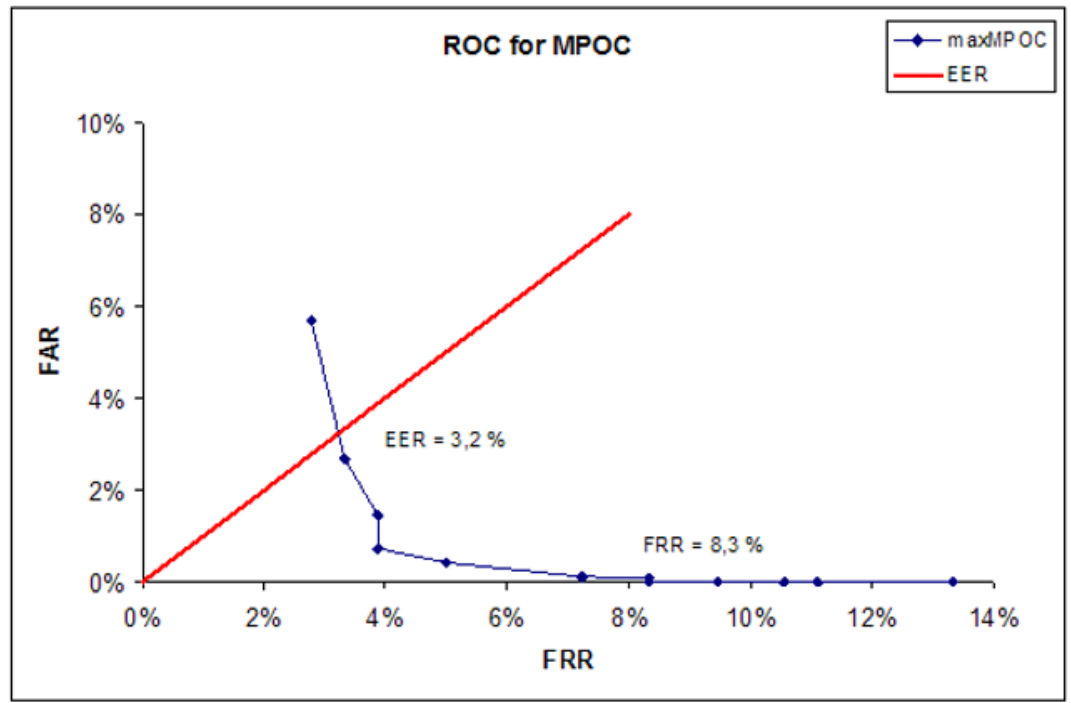

Figure 9. Receiver operating characteristic $(\mathrm{ROC})$ curve for comparing criteria of MPOC.

Comparative criteria maxMPOC for $\mathrm{FAR}=0, \mathrm{FRR}=8.3 \%$ can recognize $91.7 \%$ of fingerprints from the same persons in threshold value of 0.26 as an equivalent for $100 \%$ refusing of fingerprints from different persons.

Tab. 2 brings the comparison of the achieved results and results of other authors. 
Table 2. Comparison of the achieved results and the results of other authors.

\begin{tabular}{|c|c|c|c|}
\hline \multirow[b]{2}{*}{ Method } & \multicolumn{2}{|c|}{ For $\mathrm{FAR}=0$} & \multirow[b]{2}{*}{ EER } \\
\hline & $\begin{array}{c}\text { Identical prints - } \\
\text { falsely rejected (FRR) }\end{array}$ & $\begin{array}{l}\text { Identical prints - } \\
\text { correctly recognized }\end{array}$ & \\
\hline $\begin{array}{l}\text { Proposed method } \\
\text { (registration and MPOC) }\end{array}$ & $8.3 \%$ & $91.7 \%$ & $3.2 \%$ \\
\hline $\begin{array}{l}\text { Mutual information } \\
\text { (see Ref. [3]) }\end{array}$ & $10.0 \%$ & $90.0 \%$ & $\begin{array}{l}\text { Author } \\
\text { does not } \\
\text { mention }\end{array}$ \\
\hline $\begin{array}{l}\text { Hong's method of minutiae } \\
\text { (see Ref. [6]) }\end{array}$ & $27.7 \%$ & $72.3 \%$ & $\begin{array}{l}\text { Author } \\
\text { does not } \\
\text { mention }\end{array}$ \\
\hline $\begin{array}{l}\text { Phase correlation of BPOC } \\
\text { (see Ref. [8]) }\end{array}$ & $2.5 \%$ & $97.5 \%$ & $1.7 \%$ \\
\hline $\begin{array}{l}\text { Phase correlation } \\
\text { (see Ref. [8]) }\end{array}$ & $18.3 \%$ & $81.7 \%$ & $6.0 \%$ \\
\hline $\begin{array}{l}\text { Comparison of minutiae } \\
\text { (see Ref. [8]) }\end{array}$ & $14.2 \%$ & $85.8 \%$ & $7.0 \%$ \\
\hline $\begin{array}{l}\text { Invariant moments } \\
\text { (see Ref. [11]) }\end{array}$ & $\begin{array}{c}\text { Author does not } \\
\text { mention }\end{array}$ & $\begin{array}{c}\text { Author does not } \\
\text { mention }\end{array}$ & $3.6 \%$ \\
\hline $\begin{array}{l}\text { Cosine distance } \\
\text { (see Ref. [11]) }\end{array}$ & $\begin{array}{c}\text { Author does not } \\
\text { mention }\end{array}$ & $\begin{array}{c}\text { Author does not } \\
\text { mention }\end{array}$ & $5.4 \%$ \\
\hline
\end{tabular}

\section{The advantages of the method}

- The ability to register incomplete, shifted, turned images of fingerprints with a changed scale.

- The ability to register fingerprints from a non-edited gray-scale image without needing to pre-process the image used to compare fingerprints via minutiae (without filtration, segmentation and thinning).

- The ability to register fingerprints of worse quality because the registration based on phase correlation uses global information about the textures instead of points' properties.

- The possibility of visual control of the registration result by showing effective areas of images of reference and tested fingerprint after its backward rotation, scale and shift adjustment.

- The possibility of consequent comparison of registered images using suitable metrics and comparison scores and so enable automated decision- making whether they come from the same finger or not.

\section{Disadvantages of the method}

- Time calculation complexity. At registration of images with a shift, scale and rotation change the space of transformation parameters grows. The proposed method is demanding from the viewpoint of calculation time, because it assumes all transformations of images (rotation, shift, scale change). To register an image it is necessary to calculate 6 fast Fourier transform (FFT), 2 phase correlation including inverse FFT, 2 logarithmicpolar processing, 1 rotation, scale change and shift of an image

- Image dimensions. As at the numeric processing the fast Fourier transform is used, the dimensions of the images being processed must be $2^{N}$.

- Limited deformations. The method is able to recover only the deformations of shift, rotation and scale change of the whole image. Other deformations and deformations of larger extent are only partially eliminated. This disadvantage can be considered unimportant if we admit that the finger position in a scanner is possible to be fixed to a certain extent and assume that the fingerprints are deformed and turned only slightly. 
These days, the concept of biometrics is frequently mentioned in connection with personal identification, ensuring security and protection of people, property and various objects or data. Reliable personal identification is critical in numerous common financial, commercial and civil applications.

The proposed method does not require large scale preprocessing, partially eliminates imprecise finger position on a scanner (shift, rotation), and partially recovers fingerprint deformations (scale change, skin elasticity) and lower quality of finger skin (very dry fingers). It enables automatic processing and evaluation without an operator's intervention.

We suppose the method, with little modifications, shall also be usable to compare other biometric images, e.g. irides, palms, feet, ears etc. The program may be modified for identification (instead of verification).

\section{References}

[1] Blázsovits G., Digital Image Processing, 2006. Available on-line: http://dip.sccg.sk/body.htm [cit. October 2007] (in Slovak)

[2] Csongrády T., Pivarčiová E., Fingerprint image registration, Informatics and automation in the process control, V. Scientific Conference (10 September 2009, Zvolen), Technical university in Zvolen, 2009, 19-25 (in Slovak)

[3] Dobeš M., Image recognition with bearing to personal identification by the fingerprints, Brno University of Technology, Faculty of Electrical Engineering and Computer Science, Brno, 2001 (in Czech)

[4] Egli A. (2009), Medical Image Registration 2D/3D (X-Ray/CT). Available on-line: informatik.unibas.ch/lehre/fs09/cs503/_Downloads/egli.pdf [cit. June 2009]

[5] Gueham M., Bouridane A., Crookes D., Automatic recognition of partial shoeprints based on phase-only correlation, IEEE IMAGE PROC, 2007, IV, 441-444, available on-line: http://ieeexplore.ieee.org/xpl/freeabs_all.jsp?arnumber=4380049

[6] Hong L., Automatic Personal Identification using Fingerprint, PhD thesis, Michigan State University, East Lansing, 1998

[7] Chen Q.S., Image Registration and its Applications in Medical Imaging, Dissertation work, Vrije Universiteit Brussel, Deconinck 1993

[8] Ito K., Nakajima H., Kobayashi K., Aoki T., Higuchi T., A Fingerprint Matching Algorithm Using Phase-Only Correlation, IEICE T FUND ELECTR, 2004, E87-A, 682-691, available on-line: http://www.aoki.ecei.tohoku.ac.jp/ research/docs/e87-a_3_682.pdf

[9] Pivarčiová E., Csongrády T., Fourier-Mellin transform - tool for image registration, APERIODIC OF SLOVAK TECHNICS AND NATURALISTS: IDEAS AND FACTS, 2009, XV, 18-22 (in Slovak)

[10] Pratt J.G., Application of the Fourier-Mellin transform to translation, rotation and scale invariant plant leaf identification. Montreal, 2000. [cit. July 2009]

Available on-line: http://digitool.library.mcgill.ca:1801/view/action/singleViewer.do?dvs=1244062994431 663

\&locale $=$ sk\&show_metadata $=$ false\&preferred_extension $=$ pdf\&search_terms $=000006662$ Gadjacency $=\mathrm{N}$

Eapplication=DIGITOOL-3\&frameld=1\&usePid1 =true\&usePid2=true

[11] Yang J.Ch. (2008), Fingerprint Authentication System. Available on-line: http://yangjucheng.chonbuk.ac.kr/ [cit. January 2009] 